\title{
Impacto de la pandemia por COVID-19 en los resultados del tratamiento de la apendicitis aguda: estudio observacional retrospectivo
}

\author{
Impact of the COVID-19 pandemic on acute appendicitis treatment \\ outcomes: a retrospective observational study \\ Mora Achaval ${ }^{1} \mathbb{D}$, Juan Pablo Pratesi ${ }^{1} \mathbb{D}$, Sofía Rapp ${ }^{1} \mathbb{D}$, Carina Chwat $^{2} \mathbb{D}$ \\ 1 Médico, residente de Cirugía general, Hospital Universitario Austral, Buenos Aires, Argentina. \\ 2 Médico, especialista en Coloproctología, Hospital Universitario Austral, Buenos Aires, Argentina.
}

\begin{abstract}
Resumen
Introducción. Debido a la pandemia por COVID-19, se decretó el aislamiento social, preventivo y obligatorio. El miedo de asistir al hospital, generado por la pandemia, pudo provocar una demora en la consulta y el retraso en el diagnóstico de pacientes con apendicitis, llevando a complicaciones como perforación, absceso, peritonitis, sepsis y muerte.
\end{abstract}

Métodos. Se realizó un análisis comparativo de los hallazgos quirúrgicos y su desenlace, en los pacientes con diagnóstico de apendicitis, previo a la pandemia y durante ella.

Resultados. No se encontró diferencia en el tiempo de la consulta, pero durante la pandemia se observó una diferencia estadísticamente significativa en las características del apéndice.

Discusión. Los hallazgos durante la pandemia muestran cuadros de apendicitis aguda más avanzados que antes de la misma, sin impactar en las complicaciones quirúrgicas y postoperatorias.

Palabras clave: COVID-19; SARS-CoV-2; pandemia; apendicitis aguda; abdomen agudo; peritonitis.

\begin{abstract}
Introduction. Due to the COVID-19 pandemic, and as social and preventive isolation was mandatory, the fear of going to the hospital generated by the pandemic, could cause a delay in the consultation and delay in the diagnosis of appendicitis, leading to complications such as perforation, abscess, peritonitis, sepsis and death.

Fecha de recibido: 05/02/2021 - Fecha de aceptación: 20/02/2021 - Fecha de publicación en línea: 06/04/2021

Correspondencia: Mora Achaval, Saraví 1450, Pilar, CP 1669, Buenos Aires, Argentina. Teléfono: +54 911 6678-4529

Correo electrónico: moraachaval@hotmail.com

Citar como: Achaval M, Pratesi JP, Rapp S, Chwat C. Impacto de la pandemia por COVID-19 en los resultados del tratamiento de la apendicitis aguda: estudio observacional retrospectivo. Rev Colomb Cir. 2021;36:487-92. https://doi.org/10.30944/20117582.854

Este es un artículo de acceso abierto bajo una Licencia Creative Commons - BY-NC-ND https://creativecommons.org/licenses/by-nc$\mathrm{nd} / 4.0 /$ deed.es
\end{abstract}


Method. A comparative analysis of the surgical findings and their outcome was performed in patients with a diagnosis of appendicitis prior to and during the pandemic.

Results. No difference was found at the time of consultation, but during the pandemic a statistically significant difference was observed in the characteristics of the appendix.

Discussion. The findings during the pandemic show more advanced acute appendicitis than before, without impacting on surgical and postoperative complications.

Keywords: COVID-19; SARS-CoV-2; pandemic; acute appendicitis; acute abdomen; peritonitis.

\section{Introducción}

La apendicitis aguda (AA) es la urgencia quirúrgica abdominal más frecuente ${ }^{1}$. El retraso en el diagnóstico puede causar complicaciones como perforación apendicular, absceso apendicular, peritonitis, sepsis y muerte ${ }^{2,3}$. Debido a la pandemia por el nuevo coronavirus (COVID-19), el día 20 de marzo de 2020 se decretó el aislamiento social, preventivo y obligatorio (ASPO) en la República Argentina ${ }^{4}$. El miedo de asistir al hospital generado por la pandemia pudo condicionar la demora desde el inicio de la sintomatología abdominal hasta el momento de la consulta ${ }^{5}, \mathrm{y}$, por ende, llevar a la presentación de cuadros más evolucionados y al desarrollo de complicaciones postoperatorias $^{3}$.

En nuestro hospital, los circuitos de circulación de los pacientes están divididos en limpios y sucios. Quienes van a cirugía programada, ingresan al quirófano con una prueba de Reacción en Cadena de la Polimerasa (RT-PCR, por sus siglas en inglés) para detección de COVID-19 negativa. A los pacientes que ingresan para una cirugía de urgencia, se le solicita la PCR, como una prueba rápida para identificar infección por el SARS-CoV-2. Al momento de realizar la cirugía, generalmente el resultado está pendiente, por lo que el procedimiento se realiza en un quirófano con presión negativa y con uso de equipo de protección tipo 3 por parte de todo el personal. El paciente permanece aislado en el posoperatorio, hasta obtener el resultado de la PCR.

El objetivo de este estudio fue evaluar el impacto de la pandemia causada por el COVID-19 sobre la forma de presentación, tratamiento y morbimortalidad del paciente con apendicitis aguda.

\section{Materiales y Métodos}

Se realizó un estudio observacional, retrospectivo, incluyendo pacientes de ambos sexos, mayores de 16 años, tratados por cuadro de abdomen agudo de etiología apendicular, entre el $1^{\circ}$ de enero de 2020 y el 16 de julio de 2020. Se asignaron los pacientes en dos grupos, de acuerdo con la fecha en la cual se realizó la cirugía, así: pre ASPO y pos ASPO (a partir del 20 de marzo de 2020).

Se registraron los datos demográficos, clasificación de ASA ${ }^{6}$, tiempo de evolución de la sintomatología previo a la consulta, presencia o no de infección por COVID-19, escala de Alvarado ${ }^{7}$, recuento de glóbulos blancos, tipo de AA, tratamiento realizado, tiempo quirúrgico, hallazgos intra-operatorios, necesidad de conversión, estadía hospitalaria, complicaciones postoperatorias, resultado de la anatomía patológica, requerimiento de reintervención quirúrgica o de reingreso hospitalario.

Se definió como "complicación posoperatoria" a toda desviación del curso posquirúrgico habitual, ocurrida dentro de los 30 primeros días después de la cirugía, clasificándose como "complicaciones menores" a aquellas incluidas dentro de las categorías Clavien Dindo 1 y 2, y como "complicaciones mayores" a las descritas en las categorías 3 y $4^{8}$. Se definió como "reingreso hospitalario" al ocurrido dentro de los 30 primeros días después de la cirugía, por cualquier causa relacionada con la cirugía. 


\section{Análisis estadístico}

Para el reporte de las variables continuas se utilizó media, rango y desviación estándar, para las variables discretas se reportó el número y los porcentajes correspondientes. Las comparaciones de parámetros continuos se realizaron mediante la prueba para muestras independientes. Para la comparación de variables categóricas se utilizó la prueba de chi cuadrado o la prueba exacta de Fisher, según fuera apropiado. Se consideró estadísticamente significativo una $\mathrm{p}<0,05$. El análisis estadístico se realizó con el programa Stata ${ }^{\circledR}$ versión 14.2 (Stata-Corp. 2015. Stata Statistical Software: Release 14. College Station, TX: StataCorp LP).

\section{Resultados}

En el periodo de estudio se observó un incremento en la incidencia de apendicitis aguda, de $28,7 \%$ antes del ASPO a 32,7 \% después del mismo. Fueron tratados 98 pacientes, con una edad media de 34 años (rango de 17-66 años), $55,1 \%(n=54)$ de sexo masculino. Previo al ASPO se operaron 43 pacientes y posterior al mismo 55 pacientes. Solo uno presentó una prueba de Reacción en Cadena de la Polimerasa PCR positiva para COVID-19, representando una incidencia del $1,8 \%$ de positividad para los pacientes operados de apendicectomía. No se encontraron diferencias estadísticamente significativas en el tiempo transcurrido desde el inicio de los síntomas hasta la consulta, teniendo una media de 45 horas pre ASPO versus 48 horas pos ASPO.

No hubo diferencias en edad, patología, ASA, tiempo quirúrgico o días de internación entre los grupos comparados. Sin embargo, se observó una diferencia en aspectos de la presentación clínica que no impactaron en la escala de Alvarado. El $85 \%$ de los pacientes tratados durante la cuarentena presentaron migración, mientras que ésta se constató en el $65 \%$ del otro grupo $(p=0,02)$. Asimismo, el $76 \%$ del grupo pos ASPO y el $56 \%$ del otro grupo presentaron anorexia $(\mathrm{p}=0,03)$.

El recuento de glóbulos blancos tendió a ser mayor en aquellos pacientes operados luego del decreto de ASPO, aunque no representó una dife- rencia estadísticamente significativa. Como parte de la evaluación prequirúrgica se realizó tomografía en el 55,8 \% ( $n=24)$ de los pacientes pre ASPO $y$ en el 50,9\% ( $n=28)$ del grupo alterno (tabla 1$)$.

Todos los pacientes diagnosticados pre ASPO fueron intervenidos quirúrgicamente mientras que, en tres de los diagnosticados durante la cuarentena, se decidió realizar tratamiento conservador por tratarse de pacientes con plastrón apendicular. No se encontró una diferencia estadísticamente significativa en el tipo de tratamiento (médico/quirúrgico) entre los grupos. Los hallazgos intraoperatorios correspondieron a apendicitis gangrenosa o gangrenosa y perforada en el $52,7 \%(n=29)$ de los pacientes operados en pandemia, mientras que antes de la pandemia el porcentaje correspondió solo al 30,2 \% ( $n=13)$, $(\mathrm{p}=0,008)$.

Todos los pacientes operados fueron abordados por vía laparoscópica. Durante la cuarentena tres pacientes requirieron conversión a cirugía abierta por imposibilidad de identificar adecuadamente la anatomía debido al proceso inflamatorio, y se realizó el doble de rafias cecales que en el grupo pre ASPO.

No se observaron diferencias estadísticamente significativas en las complicaciones quirúrgicas o posoperatorias. El 11,6 \% (n=5) de los pacientes pre ASPO presentaron tres complicaciones mayores y dos menores, las complicaciones mayores incluyeron una paciente con una colección abdominal con requerimiento de drenaje percutáneo, y dos pacientes con requerimiento de colectomía derecha e ileostomía de protección por fístula del muñón apendicular. El 7,2 \% (n=4) de los pacientes pos ASPO presentaron complicaciones, dos menores en pacientes con antibioticoterapia prolongada y dos pacientes con complicaciones mayores, consistentes en drenaje percutáneo por colección abdominal y colectomía derecha por fístula del muñón (tabla 2).

El tiempo de estadía hospitalaria, las reintervenciones quirúrgicas o el reingreso de los pacientes, no presentaron diferencias estadísticamente significativas. No ocurrió mortalidad en el grupo de estudio. 
Tabla 1. Diferencias preoperatorias de los pacientes con apendicitis aguda operados antes y después del aislamiento social, preventivo y obligatorio (ASPO)

\begin{tabular}{|c|c|c|c|c|}
\hline & & $\begin{array}{c}\text { Pre ASPO } \\
n=43(\%)\end{array}$ & $\begin{array}{c}\text { Pos ASPO } \\
n=55(\%)\end{array}$ & $\mathbf{p}$ \\
\hline Edad (años) & & 34 & 34 & 0,93 \\
\hline Clasificación de $\mathrm{ASA}^{*}$ & $\begin{array}{l}1 \\
2 \\
3\end{array}$ & $\begin{array}{c}26(60 \%) \\
17(40 \%) \\
0(0 \%)\end{array}$ & $\begin{array}{c}29(56 \%) \\
22(42 \%) \\
1(2 \%)\end{array}$ & 0,62 \\
\hline $\begin{array}{l}\text { Tiempo de evolución ** (horas), } \\
\text { media (DE) }\end{array}$ & & $45(36)$ & $48(51)$ & 0,76 \\
\hline Glóbulos blancos, media (DE) & & $14.058(4081)$ & $15.819(5267)$ & 0,07 \\
\hline $\begin{array}{l}\text { Puntaje en la Escala de } \\
\text { Alvarado, media (DE) }\end{array}$ & & $7(2)$ & $8(2)$ & 0,12 \\
\hline Dolor en fosa ilíaca derecha & & $42(98 \%)$ & $55(100 \%)$ & 0,26 \\
\hline Reacción peritoneal & & $33(77 \%)$ & $43(78 \%)$ & 0,87 \\
\hline Migración & & $28(65 \%)$ & $47(87 \%)$ & 0,018 \\
\hline Náuseas o vómito & & $31(72 \%)$ & $38(69 \%)$ & 0,75 \\
\hline Anorexia & & $24(56 \%)$ & $42(76 \%)$ & 0,031 \\
\hline Fiebre & & $22(51 \%)$ & $34(62 \%)$ & 0,29 \\
\hline $\begin{array}{l}\text { Leucocitosis mayor de } \\
10.000 \mathrm{cel} / \mathrm{mm}^{3}\end{array}$ & & $35(81 \%)$ & $48(87 \%)$ & 0,42 \\
\hline Neutrofilia mayor de $75 \%$ & & $33(77 \%)$ & $47(85 \%)$ & 0,27 \\
\hline Tomografía confirma diagnóstico & & $24(55 \%)$ & $28(50 \%)$ & 0,84 \\
\hline
\end{tabular}

* ASA: American Society of Anesthesiologist; DE: desviación estándar.

** Tiempo de evolución= tiempo desde el inicio de síntomas hasta la consulta.

Tabla 2. Diferencias en el tratamiento de los pacientes con apendicitis aguda antes y después del aislamiento social, preventivo y obligatorio (ASPO)

\begin{tabular}{|c|c|c|c|c|}
\hline & & $\begin{array}{c}\text { Pre ASPO } \\
n=43(\%)\end{array}$ & $\begin{array}{c}\text { Pos ASPO } \\
n=55(\%)\end{array}$ & $\mathbf{p}$ \\
\hline \multirow{2}{*}{ Tratamiento } & Médico & $0(0 \%)$ & $3(5 \%)$ & \multirow{2}{*}{0,12} \\
\hline & Quirúrgico & $43(100 \%)$ & $52(95 \%)$ & \\
\hline \multirow[t]{2}{*}{$\begin{array}{l}\text { Tiempo quirúrgico, minutos } \\
\text { mediana (DE) }\end{array}$} & & $70(22)$ & $78(42)$ & 0,27 \\
\hline & Flegmonoso & $30(70 \%)$ & $23(42 \%)$ & \multirow[b]{2}{*}{0,008} \\
\hline Hallazgos & $\begin{array}{l}\text { Gangrenoso } \\
\text { Plastrón }\end{array}$ & $\begin{array}{c}13(30 \%) \\
0(0 \%)\end{array}$ & $\begin{array}{c}29(52 \%) \\
3(5 \%)\end{array}$ & \\
\hline Peritonitis & & $10(23 \%)$ & $12(23 \%)$ & 0,98 \\
\hline Rafia & & $1(2 \%)$ & $2(4 \%)$ & 0,67 \\
\hline Conversión a cirugía abierta & & $0(0 \%)$ & $3(6 \%)$ & 0,11 \\
\hline \multirow{2}{*}{ Complicaciones posoperatorias } & Mayores & $3(7 \%)$ & $2(3 \%)$ & \multirow{2}{*}{0,2} \\
\hline & Menores & $2(4 \%)$ & $2(3 \%)$ & \\
\hline Internación, días media (DE) & & $2(2)$ & $3(2)$ & 0,29 \\
\hline Reintervención & & $2(5 \%)$ & $1(2 \%)$ & 0,45 \\
\hline \multirow[t]{2}{*}{ Reingreso } & & $3(7 \%)$ & $2(4 \%)$ & 0,46 \\
\hline & Apendicitis aguda & $42(98 \%)$ & $52(100 \%)$ & \multirow[b]{2}{*}{0,13} \\
\hline Anatomía patológica & $\begin{array}{l}\text { Tumor neuro } \\
\text { endocrino }\end{array}$ & $1(2 \%)$ & $0(0 \%)$ & \\
\hline
\end{tabular}

*DE: desviación estándar 


\section{Discusión}

En países como el estado de Israel, se observó una disminución de las consultas por apendicitis aguda durante la pandemia, probablemente debido al aislamiento ordenado por el gobierno, y al miedo por parte de los pacientes de asistir a una consulta médica ${ }^{9}$. La incidencia de apendicitis en los pacientes con abdomen agudo quirúrgico de nuestra institución no se modificó con el inicio del ASPO, posiblemente debido a la difusión en redes sociales y canales institucionales de comunicación fomentando la consulta temprana a pesar de la pandemia.

$\mathrm{Al}$ igual que lo observado por Velayos et al. ${ }^{10}$, en este estudio no se encontró que el tiempo desde el inicio de la sintomatología hasta la consulta por urgencias se hubiera modificado con la pandemia, pero esto puede deberse a un inadecuado registro del tiempo de evolución del cuadro debido a que es un estudio retrospectivo. Sin embargo, si constatamos que los hallazgos intraoperatorios durante la pandemia mostraron un cuadro inflamatorio mayor, registrando más apendicitis gangrenosas o perforadas y peritonitis, sugiriendo un mayor tiempo de evolución del cuadro clínico. La conversión a cirugía abierta en tres casos y el tratamiento conservador en tres pacientes debido a plastrón apendicular, también sugiere una demora en el diagnóstico.

Dreyfuss demostró una diferencia estadísticamente significativa en el tiempo de la consulta, siendo mayor en el grupo pos ASPO, y asociándolo con una mayor incidencia de complicaciones postoperatorias $^{5}$. En este estudio no se encontró variación en las complicaciones posoperatorias entre los dos grupos.

A diferencia de Suwanwongse et al. ${ }^{11}$, quienes en el caso de un paciente con COVID-19 optaron por tratamiento médico, nosotros preferimos el tratamiento quirúrgico, realizando una apendicectomía laparoscópica. Se tomó esta decisión por tratarse de un paciente con sintomatología leve y sin comorbilidades. La paciente cursó con un posoperatorio sin complicaciones, a las 48 horas se le otorgó el egreso hospitalario y cumplió el aislamiento en su domicilio.
Independientemente del resultado de la PCR para COVID-19, durante la pandemia muchos cirujanos decidieron tratar médicamente a los pacientes con apendicitis ${ }^{11}$. En nuestra institución, las guías de manejo de la apendicitis aguda no se modificaron, y en este estudio, solo tres pacientes recibieron tratamiento no quirúrgico, debido al tiempo de evolución de la enfermedad y el hallazgo tomográfico compatible con plastrón apendicular. Todos los pacientes operados fueron abordados por vía laparoscópica, a diferencia de lo recomendado por el grupo Had Appendicitis and Resolved/ Recurred Emergency Morbidity/Mortality (HAREM, por sus siglas en inglés), que sugiere limitar el abordaje laparoscópico durante la pandemia debido a la potencial transmisión del virus por aerosoles ${ }^{12}$.

\section{Conclusión}

La apendicitis aguda continúa siendo la causa de abdomen agudo quirúrgico más frecuente durante la pandemia. Al comparar los pacientes tratados en el periodo pre ASPO, con los tratados en el periodo posterior, los de este último grupo, han tenido casos de apendicitis aguda más avanzada, tipo gangrenosa y perforada e incluso plastrones apendiculares, pero sin presentar mayores complicaciones quirúrgicas o posoperatorias.

\section{Cumplimiento de normas éticas}

Consentimiento informado: Teniendo en cuenta que los datos analizados en este estudio son parte de la práctica asistencial y quirúrgica habitual de los pacientes, sin ningún procedimiento adicional y sabiendo que previo a su internación firmaron un consentimiento informado en el cual dieron su autorización para la utilización de sus datos con fines de investigación, no se solicitó la firma de un consentimiento adicional. El Comité de Evaluación Institucional autorizó la confección y análisis de la base de datos, dando su aprobación con $\mathrm{N}^{\circ}$ B20-011.

Conflictos de interés: Los autores declaran que no presentan conflictos de interés.

Fuente de financiación: la presente investigación no recibió ayudas específicas provenientes del sector público, comercial o de entidades sin ánimo de lucro.

Contribución de los autores:

Concepción y diseño del estudio: Mora Achaval, Juan Pablo Pratesi, Sofía Rapp, Carina Chwat. 
Adquisición de datos: Mora Achaval, Juan Pablo Pratesi, Sofía Rapp, Carina Chwat.

Análisis e interpretación de datos: Mora Achaval, Juan Pablo Pratesi, Sofía Rapp, Carina Chwat.

Redacción del manuscrito: Mora Achaval, Juan Pablo Pratesi, Sofía Rapp, Carina Chwat.

Revisión crítica: Mora Achaval, Juan Pablo Pratesi, Sofía Rapp, Carina Chwat.

\section{Referencias}

1 Körner H, Söndenaa K, Söreide JA, Andersen E, Nysted A, Lende $\mathrm{TH}$, et al., Incidence of acute nonperforated and perforated appendicitis: age-specific and sexspecific analysis. World J Surg. 1997;21:313-7 https://doi.org/10.1007/s002689900235

2 Sanabria Á, Domínguez LC, Vega V, Osorio C, Serna A, Bermúdez C. Tiempo de evolución de la apendicitis y riesgo de perforación. Rev Colomb Cir. 2013;28:24-30.

3 Kearney D, Cahill RA, O’Brien E, Kirwan WO, Redmond HP. Influence of delays on perforation risk in adults with acute appendicitis. Dis Colon Rectum. 2008;51:1823-7. https://doi.org/10.1007/s10350-008-9373-6

4 Boletín Oficial de la República Argentina, Decreto de Necesidad y Urgencia 297/2020. 20 de marzo de 2020. Fecha de consulta: 24 de enero de 2021. Disponible en: https://www.boletinoficial.gob.ar/detalleAviso/ primera/227042/20200320

5 Dreifuss NH, Schlottmann F, Sadava EE, Rotholtz NA. Acute appendicitis does not quarantine: surgical outcomes of laparoscopic appendectomy in COVID-19 times. Br J Surg. 2020;107:e368-9. https://doi.org/10.1002/bjs.11806

6 Junger A, Engel J, Quinzio L, Banzhaf A, Jost A, Risikoindizes $\mathrm{H}$, Scoring-systeme und prognostische modelle in der anästhesie und intensivmedizin. teil i: anästhesie [Risk predictors, scoring systems and prognostic models in anesthesia and intensive care. Part I: anesthesia]. Anasthesiol Intensivmed Notfallmed Schmerzther. 2002;37:520-7.

https://doi.org/10.1055/s-2002-33769

7 Maghrebi H, Maghraoui H, Makni A, Sebei A, Fredj SB, Mrabet A, et al., Intérêt du score d'Alvarado dans le diagnostic des appendicites aigües [Role of the Alvarado score in the diagnosis of acute appendicitis]. Pan Afr Med J. 2018;29:56. https://doi.org/10.11604/pamj.2018.29.56.14011

8 Bolliger M, Kroehnert JA, Molineus F, Kandioler D, Schindl M, Riss P. Experiences with the standardized classification of surgical complications (Clavien-Dindo) in general surgery patients. Eur Surg. 2018;50:256-61. https://doi.org/10.1007/s10353-018-0551-z

9 Tankel J, Keinan A, Blich O, Koussa M, Helou B, Shay S, et al., The decreasing incidence of acute appendicitis during COVID-19: a retrospective multi-center study. World J Surg. 2020;44:2458-63.

https://doi.org/10.1007/s00268-020-05599-8

10 Velayos M, Muñoz-Serrano AJ, Estefanía-Fernández K, Sarmiento-Caldas MC, Moratilla-Lapeña L, López-Santamaría M, et al., Influencia de la pandemia por coronavirus (SARS-Cov-2) en la apendicitis aguda An Pediatr (Barc). 2020;93:118-22.

https://doi.org/10.1016/j.anpedi.2020.04.022

11 Suwanwongse K, Shabarek N. Successful conservative management of acute appendicitis in a coronavirus disease 2019 (COVID-19) Patient. Cureus. 2020;12:e7834. https://doi.org/10.7759/cureus.7834

12 HAREM Steering Group. The HAREM (Had Appendicitis and Resolved/Recurred Emergency Morbidity/Mortality) Study. Br J Surg. 2020;107:e257. https://doi.org/10.1002/bjs.11711 